\title{
Structure formation in binary colloids
}

\author{
I. Varga, ${ }^{1}$ F. Kun, ${ }^{1, *}$ and K. F. Pál ${ }^{2}$ \\ ${ }^{1}$ Department of Theoretical Physics, University of Debrecen, P.O. Box 5, H-4010 Debrecen, Hungary \\ ${ }^{2}$ Institute of Nuclear Research (ATOMKI), P.O. Box 51, H-4001 Debrecen, Hungary
}

(Received 22 October 2003; published 25 March 2004)

\begin{abstract}
A theoretical study of the structure formation observed very recently [W. D. Ristenpart, I. A. Aksay, and D. A. Saville, Phys. Rev. Lett. 90, 128303 (2003)] in binary colloids is presented. In our model solely the dipole-dipole interaction of the particles is considered, electrohydrodynamic effects are excluded. Based on molecular dynamics simulations and analytic calculations we show that the total concentration of the particles, the relative concentration, and the relative dipole moment of the components determine the structure of the colloid. At low concentrations the kinetic aggregation of particles results in fractal structures which show a crossover behavior when increasing the concentration. At high concentration various lattice structures are obtained in a good agreement with experiments.
\end{abstract}

DOI: 10.1103/PhysRevE.69.030501

PACS number(s): 83.10.Pp, 82.70.Dd, 41.20.-q, 61.46. $+\mathrm{w}$

Recently, the structure formation in a monolayer of colloidal particles under an external electric or magnetic field attracted considerable scientific interest. Investigations have been performed with several different types of particles subjected to a constant or time dependent external fields. While in the absence of an external field chains, rings and fractal clusters have been observed with a temperature dependent fractal dimension [1-11], in the presence of an external field crystal structures emerged [12-14]. It was demonstrated that by appropriate adjusting of the external field all the planar lattice structures can be produced in a magnetic monolayer [12], which is of high practical importance and also addresses essential problems of statistical physics such as the study of phase transitions between different morphologies and the phenomenon of melting in two dimensions [12-14].

Binary colloids, i.e., suspensions composed of two sorts of particles of the same size have been found very recently to produce novel structures when subjected to an ac electric field [15]. Experiments performed with different frequencies and volume fractions of the components revealed a rich variety of self-assembling structures in certain frequency ranges: at frequencies where the effective particle interaction is repulsive a triangular lattice of particles (Wigner crystal) was observed [15]. At frequencies, however, where different types of particles attract each other, aggregation and crystallization occurred: at low concentrations strings and rings of alternating particles emerged while at higher concentrations various lattice structures were obtained [15]. It was found that the morphology also depends on the relative concentration of the particles, i.e., if one type of particles has a sizable excess flowerlike clusters are formed with one type of particle in the middle surrounded by five to six particles of the other type. In regions of 1:1 or 1:2 relative concentrations particles crystallize into binary honeycomb or square-packed superlattices. It has been shown that electrohydrodynamic (EHD) flow is alone not enough to understand the obtained structure formation but the dipolar interaction of the particles arising due to their induced dipole moment might have a

*Electronic address: feri@dtp.atomki.hu governing role to produce the observed structures [15]. Similar patterns have also been observed in one-component dipolar ensembles under a high external field [16].

In this paper we present a theoretical study of the novel structure formation in binary colloids observed in Ref. [15]. Our goal is to understand the effect of dipolar forces on the structure formation, furthermore, to identify the governing parameters of the system and explore all possible aggregate and crystalline morphologies determining the corresponding parameter regime of their occurrence.

In the experiment the particles are settled down to the bottom plate of a container and an ac electric field is imposed perpendicular to the bottom. Under the influence of the external field the particles attain a dipole moment parallel to the field, however, due to the strong field dependence of the polarizabilities of the particles, the magnitude and the actual direction can be different for particles made of different materials, as it was pointed out by Ref. [15]. In our model an ensemble of $N$ particles of radius $R$ is considered in a square box of side length $L$. Pointlike dipole moments are assumed to be placed in the middle of the particles. For simplicity, the dipole moment of the particles is fixed during the time evolution of the system to be perpendicular to the plane of motion pointing either upward (particle type I) or downward (particle type II), which represents the two different material properties of the particles. This assumption implies that in the model only that range of the external field imposed in the experiments is considered, where different particles attract each other. The particles are considered to be suspended in an electromagnetically passive liquid so that EHD effects are completely excluded in the model. The liquid only exerts a friction force (Stokes force) on the particles which move under the action of dipole-dipole forces. When particles touch each other during their motion we introduce a repulsive force of the form of Hertz contact to prevent the overlap $[8,9]$. After generating an initial configuration with random particle positions in the simulation box, and fixing the dipole moments according to the different material's properties, the time evolution of the system is followed by solving numerically the equation of motion of particles for the two translational degrees of freedom with periodic boundary conditions 


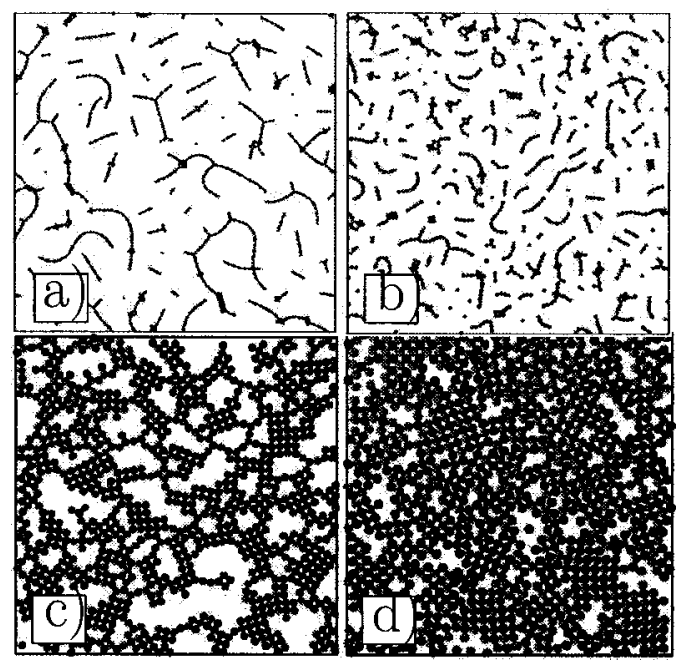

FIG. 1. (Color online) The aggregation of dipoles with fixed $\phi_{r}=1, \quad \mu_{r}=1$ at different concentrations: (a) $\phi=0.075$, (b) $\phi=0.125$, (c) $\phi=0.5$, and (d) $\phi=0.70$.

(molecular dynamics simulation). The periodic boundary condition implies a cutoff of the long range interaction at a distance $L / 2$. In the model the motion of particles is deterministic, i.e., no stochastic forces are taken into account due to the high particle mass which hinders thermal motion [15]. For details of our simulation techniques see Refs. [8,9]. A model system of $N$ particles consists of $N_{1}$ and $N_{2}$ particles of dipole moment $\mu_{1}$ pointing upward $(+)$ and dipole moment $\mu_{2}$ pointing downward $(-)$, respectively. The concentration of particles $\phi$ is defined as the coverage $\phi$ $=N R^{2} \pi / L^{2}$. The partial concentration of the two components $\phi_{1}, \phi_{2}$ can be defined analogously whose ratio provides their relative concentration $\phi_{r}=\phi_{2} / \phi_{1}$. In the model the magnitudes $\mu_{1}, \mu_{2}$ of dipole moments can be freely varied which captures up to some extent the effect of frequency tuning of the experiment. For simplicity, we fix $\mu_{1}$ and vary the ratio $\mu_{r}=\mu_{2} / \mu_{1}$. Two particles with parallel dipole moments exert a force onto each other which is isotropic (central), it always falls in the plane of motion parallel to the line connecting the two particles. The force has a $1 / r^{3}$ dependence on the separation $r$ of the particles and it is repulsive for particles of the same type and attractive for different ones. It is interesting to note that the system is rather similar to an ensemble of charges where the interaction force decreases faster than the Coulomb force.

Simulations have been carried out varying the three parameters of the model $\phi, \phi_{r}$, and $\mu_{r}$ in a broad range. For each simulation 1000 particles were used so that $\phi$ and $\phi_{r}$ were controlled by varying the side length $L$ of the simulation box. Figure 1 shows simulation results obtained at different concentrations $\phi$ fixing the relative concentration $\phi_{r}$ $=1$ and the relative dipole moment $\mu_{r}=1$. It can be seen that at low concentrations the particles undergo a kinetic aggregation process and form chains and rings of alternating types of particles [Figs. 1(a) and (b)]. Isotropic interparticle forces result in anisotropic clusters since at low concentrations cluster structures are determined by the tendency that particles with parallel (oppositely) directed dipole moments

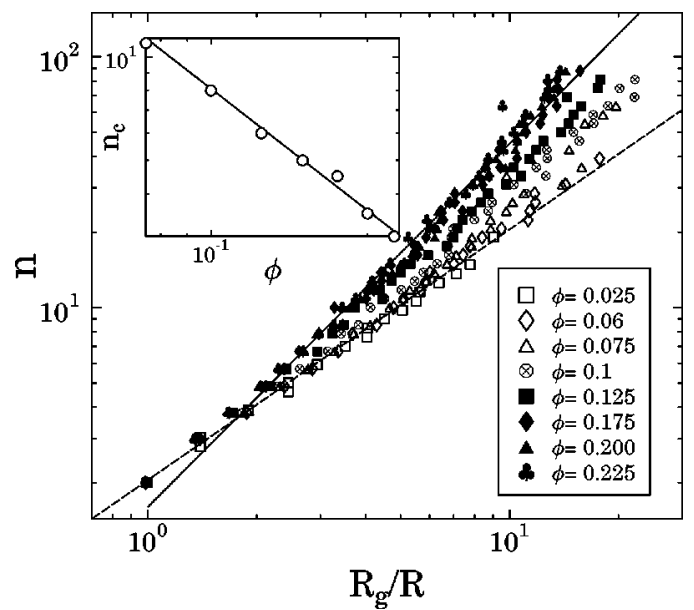

FIG. 2. The number of particles, $n$, of clusters as a function of the radius of gyration $R_{g}$. The dashed and continuous straight lines have slope 1 and 1.45 , respectively. The crossover value $n_{c}$ was obtained as the crossing point of the straight lines fitted to the two regimes of different slopes. The inset shows $n_{c}(\phi)$ where a straight line of slope $1.18 \pm 0.05$ was fitted to the data.

try to maximize (minimize) their distance. Hence, at low concentrations the chain is the dominating morphology, however, it can be shown analytically that for an even number of alternating particles the ring structure is energetically favorable [17]. [A ring can be observed in the upper part of Fig. 1(b).] More compact structures of lower energy are not accessible for the particle system in the low concentration limit since the clusters get trapped in local energy minima provided by chains and rings due to the hindered thermal motion. The length of alternating chains is limited, when the chain length becomes comparable to the average distance of chains, aggregation can occur not only at chain ends but also at internal particles resulting in branching and more compact structures. In order to characterize the structure of growing clusters we calculated the radius of gyration $R_{g}^{2}(n)=1 / n(n$ -1) $\sum_{i \neq j}\left(\vec{r}_{i}-\vec{r}_{j}\right)^{2}$ for each cluster during the time evolution of the system and averaged over clusters of the same size $n$. In Fig. 2 the cluster size $n$ is plotted as a function of the averaged gyration radius $R_{g}$ on a double logarithmic plot for several different concentrations. It can be observed in the figure that for each concentration $n\left(R_{g}\right)$ is composed of two parts of power law functional form $n \sim R_{g}^{\alpha}$ : for small clusters $n\left(R_{g}\right)$ can be well fitted by a power law of exponent close to 1 indicating chainlike structures. At a certain chain length $n_{c}(\phi)$, however, a crossover occurs into more compact branching structures characterized by a higher exponent of $R_{g}, \alpha=1.45 \pm 0.05$ was determined from simulations for $\mu_{r}$ $=1$ and $\phi_{r}=1$. It follows from our argument that the crossover chain length $n_{c}$ has a power law dependence on the concentration, i.e., $n_{c} \sim \phi^{-\beta}$ with an exponent $\beta=1.0$. The numerical result is presented in the inset of Fig. 2 where $\beta=1.18 \pm 0.05$ was obtained in a reasonable agreement with the analytic prediction. Simulations also showed that the exponent $\alpha$ characterizing the structure of growing aggregates does not depend on $\phi$ until $\phi<\phi^{*} \approx 0.25$. It is important to emphasize, however, that $\phi_{r}$ and $\mu_{r}$ have a substantial effect 


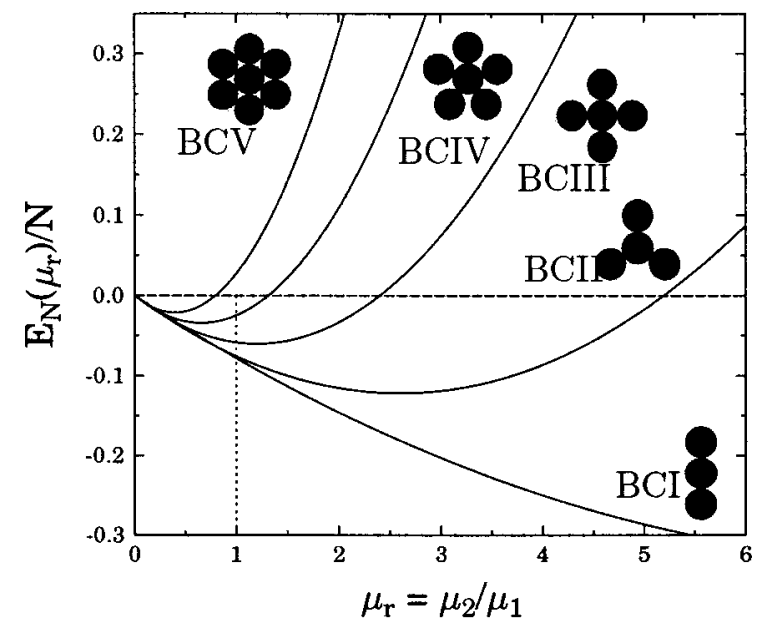

FIG. 3. (Color online) $E_{N}\left(\mu_{r}\right) / N$ from Eq. (1) for $N=3,4,5,6$, and 7. For clarity, the corresponding particle configurations are also presented.

on the aggregation process which will be explored in its entire complexity elsewhere [17].

Increasing the concentration above $\phi^{*}$ the morphology of clusters changes drastically; crystallites of square-packed structure are formed which then aggregate into large clusters. Fig. 1(c) shows simulation results obtained at $\phi=0.5$ where a network of crystallites can be observed. At higher concentrations the entire system self-assembles into a square-packed lattice of alternating particles as in Fig. 1(d).

In order to understand the formation of crystal structures in binary colloids and explore possible lattice morphologies beyond the square-packed one, we calculated the energy of basic particle configurations from which lattices can be built up. The basic configurations (bc) are composed of a particle of type I (with dipole moment $\mu_{1}$ ) surrounded by 2 (BCI), 3 (BCII), 4 (BCIII), 5 (BCIV), or 6 (BCV) particles of type II (with dipole moment $\mu_{2}$ ) placed at the energetically most favorable locations. In order to simply cover also cases where the different types of particles are interchanged, the energy of BCs was calculated as a function of $\mu_{r}=\mu_{2} / \mu_{1}$ fixing the value of $\mu_{1}=1$,

$$
E_{N}\left(\mu_{r}\right)=\frac{\mu_{1}^{2}}{d^{3}}\left[\frac{\mu_{r}^{2}}{8} \sum_{k=1}^{N-2} \frac{N-k-1}{\sin ^{3}(k \beta)}-\mu_{r}(N-1)\right],
$$

where $\beta=\pi /(N-1)$ and the particle number takes the values $N=3,4,5,6$, and 7 . The energy divided by the particle number $E_{N}\left(\mu_{r}\right) / N$ is presented in Fig. 3 as a function of $\mu_{r}$ for the possible values of $N$. It can be seen in the figure that all the BCs are stable (have a negative energy) only in certain $\mu_{r}$ ranges between 0 and a $\mu_{r}^{\max }(N)$ since with increasing $\mu_{r}$ the attraction exerted by the central particle is not enough to compensate the mutual repulsion of the surrounding ones. Such configurations, where the central particle is surrounded by particles of smaller dipole moment, i.e., $\mu_{r}$ $<1$ in Fig. 1, are always stable except for BCV because $\mu_{r}^{\max }(7) \approx 0.8$ falls below 1 . The upper bounds can be determined exactly from Eq. (1) as $\mu_{r}^{\max }(N) \approx 16,5.2,2.4,1.3$, and

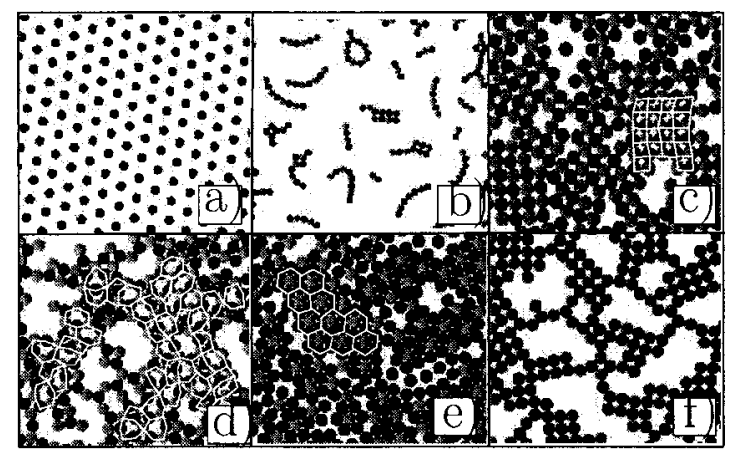

FIG. 4. (Color online) Aggregate and crystalline morphologies obtained by simulations. (a) Triangular lattice of a one-component system, (b) chains and rings, (c) square lattice, (d) honeycomb I, (e) honeycomb II, (f) superstructure. For visualization smaller pieces of the simulation box are cut out and magnified.

0.8 was obtained for $N=3,4,5,6$, and 7, respectively. Repeating the above basic structures with alternating particles various structures can be built up, i.e., BCI results in chains or rings, BCII gives rise to a honeycomb lattice in which both types of particles have three neighbors of the other type, and BCIII leads to the square lattice. BCIV results only in flowerlike structures, no lattice can be constructed since the plane cannot be covered by regular pentagons. Moreover, BCV forms the basis of a special type of honeycomb lattice, in which particles of the larger dipole moment have six neighbors of the smaller one but particles of the smaller moment have three neighbors of both types. The overall morphology attained by the entire particle system at a given value of $\mu_{r}$ is not necessarily based on the energetically most favorable basic configuration. In spite of the isotropic particle-particle interaction the cluster-cluster interaction is highly anisotropic which can result in trapping the particles into local energy minima in configuration space, especially when thermal motion is hindered by the relatively large particle mass. Hence, the relative dipole moment $\mu_{r}$, the total concentration $\phi$, and the relative concentration $\phi_{r}$ of components together determine the final structure. Based on the energy of basic configurations, Eq. (1), and Fig. 3, it is possible to determine analytically regions of $\phi, \phi_{r}$, and $\mu_{r}$ which are needed to obtain a certain lattice structure or aggregate morphology. Figure 4 provides an overview of possible structures obtained by simulations varying $\phi, \phi_{r}$, and $\mu_{r}$.

If one of the components has a much larger concentration than the other one (for instance, $\phi_{1} \gg \phi_{2}$ ), due to the repulsive interaction of the identical particles a triangular lattice is formed with some binary islands. The limiting case when only one of the components is present is shown in Fig. 4(a) where a regular triangular lattice is observed. The simplest basic configuration BCI gives rise to chain and ring conformations which occur at $\mu_{r}=1, \phi_{r}=1$, in the concentration range $\phi<\phi^{*} \approx 0.25$ with a crossover into more compact morphologies as discussed above. For an even number of particles the ring is energetically more favorable than the chain configuration. In spite of this, chains cannot close to form rings because the bending would require energy. Rings appear when two nearby chains merge which occur most fre- 
quently at concentrations $\phi \approx 0.125$, see Fig. 4(b). Similar chain and string formation of alternating particles has been reported in Ref. [15].

At high concentrations lattice structures can be obtained, however, concentrations too close to the highest coverage $(\phi \approx 0.9)$ are disadvantageous because they prevent particle motion and result in freezing into local energy minima. Hence, the best square lattice structures can be achieved at $\phi \approx 0.65-0.75$. This type of lattice contains the same amount of both types of particles so that $\phi_{r}=1$ and $\mu_{r}=1$ follows, see Fig. 4(c). The parameter regime providing square lattice agrees well with the experimental observation of Ref. [15]. The first type of honeycomb lattice (honeycomb I) where each particle has three neighbors of the other type has not been observed experimentally. However, our analytic calculations showed that energetically it can be favorable for the system in a certain parameter range. An example is presented in Fig. 4(d) which was obtained at $\phi=0.5$ and $\phi_{r}=1$. For clarity, some plaquettes of the lattice are highlighted in the figure. The basis of this structure is BCII which is always stable for $\mu_{r}<1$, i.e., when a larger dipole is surrounded by three smaller ones. However, to make the inverse configuration more favorable, $\mu_{r}$ has to be increased above the stability limit of BCIII [above $\mu_{r}^{\max }(5) \approx 2.4$ ], otherwise, the system ends up in a square-packed structure. $\mu_{r} \approx 2.5$ proved to be an excellent choice numerically, see Fig. 4(d). If the magnitude of the two dipole moments are different $\left(\mu_{r} \neq 1\right)$ concentration fluctuations easily lead to the formation of the second type of honeycomb lattice (honeycomb II), which has a hexagonal-closed-packed structure, where particles of the larger dipole moment have six neighbors of the other type and the ones with smaller dipole moment have three of both types. The highest portion of the system was found to crystallize into honeycomb II at $\phi \approx 0.80-0.82$ with the ratio of $\phi_{r}=2$ of the components and with high enough asymmetry of the magnitude of dipole moments $\mu_{r} \approx 2.5$, which is needed to prevent the system to crystallize locally into the square-packed structure. The corresponding simulation results can be seen in Fig. 4(e), which is in a nice agreement with the experiments [15]. If the relative dipole moment $\mu_{r}$ is even higher $\left(\mu_{r}>2.5\right)$ but the concentration and the relative concentration do not favor the emergence of honeycomb II, so-called superstructures can be observed in the colloid. These structures do not have ordered crystalline morphology, instead they are characterized by long straight binary chains which connect disordered or small crystalline island as in Fig. 4(f).

In summary, we proposed a simple model of a binary monolayer of dipolar particles to explore the effect of the induced dipole-dipole interaction in the intriguing structure formation observed recently [15]. Varying the three parameters of the model, i.e., the total concentration of the particles $\phi$, the relative concentration $\phi_{r}$, and the relative dipole moment $\mu_{r}$ of the components, a rich variety of structures were obtained in satisfactory agreement with the experimental findings [15]. The simplicity of the model demonstrates that the main qualitative features of the structure formation observed are determined by the induced dipole-dipole interaction. Further theoretical studies are in progress and further experiments are proposed.

This work was supported by the OTKA Projects Nos. T037212 and M041537. F. K. was supported by the Research Contract No. FKFP 0118/2001 and by the György Békési Foundation of the Hungarian Academy of Sciences.
[1] A.T. Skjeltorp, Phys. Rev. Lett. 51, 2306 (1983).

[2] J.M. Tavares, J.J. Weis, and M.M.T. da Gama, Phys. Rev. E 65, 061201 (2002).

[3] J.J. Weis, J.M. Tavares, and M.M.T. da Gama, J. Phys.: Condens. Matter 14, 9171 (2002).

[4] P.I.C. Teixeira, J.M. Tavares, and M.M. Telo da Gama, J. Phys.: Condens. Matter 12, R411 (2000).

[5] A. Ghazali and J.C. Lévy, Phys. Rev. B 67, 064409 (2003).

[6] J.Y. Huang and P.Y. Lai, Physica A 281, 105 (2000).

[7] E.M. Furst and A.P. Gast, Phys. Rev. E 61, 6732 (2000).

[8] W. Wen, F. Kun, K.F. Pál, D.W. Zheng, and K.N. Tu, Phys. Rev. E 59, R4758 (1999).

[9] F. Kun, K.F. Pál, W. Wen, and K.N. Tu, Phys. Lett. A 277, 287 (2000); F. Kun, W. Wen, K.F. Pál, and K.N. Tu, Phys. Rev. E
64, 061503 (2001).

[10] D.L. Blair and A. Kudrolli, Phys. Rev. E 67, 021302 (2003).

[11] J. Strambaugh, D.P. Lathrop, E. Ott, and W. Losert, Phys. Rev. E 68, 026207 (2003).

[12] W. Wen, L. Zhang, and P. Sheng, Phys. Rev. Lett. 85, 5464 (2000).

[13] S. Yeh, M. Seul, and B.L. Shralman, Nature (London) 386, 57 (1997).

[14] Y. Choi, K. Kim, and H.K. Pak, Physica A 281, 99 (2000).

[15] W.D. Ristenpart, I.A. Aksay, and D.A. Saville, Phys. Rev. Lett. 90, 128303 (2003).

[16] J.J. Weis, Mol. Phys. 100, 579 (2002).

[17] I. Varga, F. Kun, and K.F. Pál (unpublished). 